\title{
Incompressible impulsive sloshing
}

\author{
Peder A. Tyvand \\ Department of Mathematical Sciences and Technology \\ Norwegian University of Life Sciences \\ 1432 Ås, Norway \\ Touvia Miloh \\ School of Mechanical Engineering \\ Tel Aviv University \\ Ramat Aviv 69978, Israel
}

\begin{abstract}
Incompressible impulsive sloshing in open containers is studied analytically to the leading order in a small-time expansion. The surface velocity and the hydrodynamic force are calculated.
\end{abstract}

\section{On the impulsive time scale}

We consider an open container that is partly filled with liquid in equilibrium with a horizontal free surface in the gravity field. The rigid container is put into forced motion at time $t=0^{+}$. The inviscid free-surface flow has three basic time scales: (i) The acoustic time scale, which is the time an acoustic wave takes to travel one unit of characteristic length. (ii) The impulsive time scale. (iii) The gravitational time scale equals the time a body takes to fall freely one unit of characteristic length.

The impulsive time scale fills the gap between the acoustic time scale and the gravitational time scale, but it lacks a general explicit definition. Impulsive flow tends to be underrated in the sloshing literature (Ibrahim 2005, Faltinsen and Timokha 2009).

The early acoustic flow does not deform the free surface significantly, but it delays the inertial impulsive flow. The incompressible impulsive flow forces the surface particles to move in the vertical direction. The gravitational flow starts after the impulsive flow has deformed the free surface. 


\section{Mathematical problem in 3D}

The fluid model is inviscid and incompressible, with potential flow starting impulsively from rest. The two horizontal translational modes of motion are sway and surge in the $x$ and $y$ directions. A material point $(X, Y, Z)$ of the rigid container experiences a forced motion $\left(X(t), Y(t), Z_{0}\right)$ given as

$$
(X(t), Y(t))=\left(X_{0}, Y_{0}\right)+H(t)\left(\left(X_{1}, Y_{1}\right) t+\left(X_{2}, Y_{2}\right) t^{2}+\ldots\right)
$$

where the normal velocity of the fluid equals that of the container at the walls. $H(t)$ is the Heaviside unit step function. We will work with two basic cases. Primarily the case of initial impulsive velocity

$$
\left(X_{1}, Y_{1}\right)=(U, V)
$$

without subsequent acceleration $\left(X_{2}=Y_{2}=0\right)$. The secondary case is the case of initial impulsive acceleration $\left(2 X_{2}, 2 Y_{2}\right)$ with zero initial velocity $\left(X_{1}=Y_{1}=0\right)$. The flow due to impulsive motion of a rigid container is given by a small-time expansion for the potential $\Phi$ and surface elevation $\eta$

$$
\begin{gathered}
\Phi(x, y, z, t)=H(t)\left(\phi_{0}(x, y, z)+t \phi_{1}(x, y, z)+t^{2} \phi_{2}(x, y, z)+\ldots\right) \\
\eta(x, y, t)=H(t)\left(t \eta_{1}(x, y)+t^{2} \eta_{2}(x, y)+\ldots\right) .
\end{gathered}
$$

The free surface is initially horizontal, with zero horizontal velocity

$$
\eta(x, y, 0)=0, \quad \Phi(x, y, 0,0)=0 .
$$

since there is no tangential force on the free surface during the impulsive start. The net pressure force on the container walls is

$$
\vec{F}(t)=\vec{F}_{-1} \delta(t)+H(t)\left(\vec{F}_{0}+t \vec{F}_{1}+. .\right),
$$

where $\delta(t)$ denotes the Dirac delta function and the subscript -1 refers to the instantaneous singular force impulse resulting from the sudden motion of the container.

The set of variables $\left(\phi_{0}, \eta_{1}, p_{-1}, \vec{F}_{-1}\right)$ for impulsive sway velocity $X_{1}$ relates to the corresponding set $\left(\phi_{1}, \eta_{2}, p_{0}, \vec{F}_{0}\right)$ for impulsive sway acceleration $2 X_{2}$ by the following transformations

$$
\frac{\phi_{1}}{2 X_{2}}=\frac{\phi_{0}}{X_{1}}, \quad \frac{\eta_{2}}{X_{2}}=\frac{\eta_{1}}{X_{1}}, \quad \frac{p_{0}}{2 X_{2}}=\frac{p_{-1}}{X_{1}}, \quad \frac{\vec{F}_{0}}{2 X_{2}}=\frac{\vec{F}_{-1}}{X_{1}} .
$$

A similar set of transformations link the solutions for impulsive surge velocity $Y_{1}$ and impulsive surge acceleration $Y_{2}$ in the $y$ direction. These transformations are only valid to the leading order in the small-time expansion. 


\section{A wedge container}

Our first problem is a rigid wedge composed of two sloping walls with angles $\pm \pi / 4$ and depth $H$, combined with two vertical end-walls at $y=0$ and $y=L$. The impulsive sway motion with velocity $U$ in the cross-wise $x$ direction produces a simple 2D flow

$$
\phi_{0}(x, z)=U\left(1-\frac{x}{H}\right) z
$$

The leading-order surface velocity is regular everywhere, given by

$$
\eta_{1}=\left.\frac{\partial \phi_{0}}{\partial z}\right|_{z=0}=U\left(1-\frac{x}{H}\right)
$$

The total net impulsive sway force on the two slopes is

$$
F_{-1 x}=-\frac{1}{3} \rho H^{2} L U=-\frac{1}{3} m U .
$$

where $m$ denotes the fluid mass within the container. The impulsive motion of the wedge container with velocity $V$ in the lengthwise $y$ direction generates a 3D flow with free-surface velocity

$$
\begin{gathered}
\eta_{1}=\left.\frac{\partial \phi_{0}}{\partial z}\right|_{z=0}=\frac{8 V}{\pi^{2}} \sum_{m=1}^{\infty} \frac{\sin \left(\left(m-\frac{1}{2}\right) \frac{\pi x}{H}\right)}{2 m-1} \\
\sum_{n=1}^{\infty} \frac{\cosh \left(\frac{\pi(y-L)}{H} \sqrt{\left(m-\frac{1}{2}\right)^{2}+\left(n-\frac{1}{2}\right)^{2}}\right)-\cosh \left(\frac{\pi y}{H} \sqrt{\left(m-\frac{1}{2}\right)^{2}+\left(n-\frac{1}{2}\right)^{2}}\right)}{\sqrt{\left(m-\frac{1}{2}\right)^{2}+\left(n-\frac{1}{2}\right)^{2}} \sinh \left(\frac{\pi L}{H} \sqrt{\left(m-\frac{1}{2}\right)^{2}+\left(n-\frac{1}{2}\right)^{2}}\right)}
\end{gathered}
$$

and has a logarithmic singularity along the waterlines for $y=0$ and $y=L$.

\section{Other container geometries}

The impulsive flow inside a rigid half-filled horizontal cylinder is analyzed. The crosswise sway motion with velocity $U$ in the $x$ direction gives the $2 \mathrm{D}$ free-surface velocity

$$
\eta_{1}=\left.\frac{\partial \phi_{0}}{\partial z}\right|_{z=0}=\left.\frac{1}{r} \frac{\partial \phi}{\partial \theta}\right|_{\theta=0}=-\frac{8 U}{\pi} \sum_{n=1}^{\infty} \frac{n}{4 n^{2}-1}\left(\frac{x}{R}\right)^{2 n-1} .
$$

We note the logarithmic singularity in the surface velocity that arises at the waterlines $x= \pm R$. The net impulsive sway force acting on the half cylinder is given by

$$
F_{-1 x}=-\frac{4}{\pi^{2}} m U
$$


The 3D surge flow field has been found, but it is very complicated.

We have also solved the 3D impulsive flow problem within a hollow vertical circular cylinder where the velocity at the cylinder walls $r=R_{1}$ and $r=R_{2}$ may have arbitrary size and direction. This example includes the earlier studies in as special cases: Chwang and Wang (1984) studied a rectangular box and a vertical cylinder. Roberts (1988) studied a rectangular box, by a different method.

\section{Concluding remarks}

Impulsive start of sloshing is vital for improving the understanding of initial conditions, since it is far from obvious how to bring a stagnant fluid into an irrotational sloshing flow by moving the container walls. All initiation of incompressible free-surface flow by forced motion of a rigid container will be impulsive, no matter how smoothly it is started. This will lead to forces that may be significantly greater than those predicted by a time-harmonic analysis. The early transient waves generated by the impulsive flow will leave their mark on all following waves and inviscid flow patterns within a finite container.

Most container geometries will generate immediate logarithmic singularities in the free-surface flow at the waterlines where the wall meets the free surface. These singularities are integrable and give finite values for the initial impulsive force. However, the later evolution of the free surface will require matched asymptotic expansions where the present solutions serve as the leading-order outer solutions.

\section{References}

A.T. Chwang and K.-H. Wang (1984) J. Fluids Engng. 106, 233-240. O.M. Faltinsen and A.N. Timokha (2009) "Sloshing". Cambridge Univ. Press, 577 pp.

R.A. Ibrahim (2005) "Liquid Sloshing Dynamics: Theory and Applications". Cambridge Univ. Press, 948 pp.

A.J. Roberts (1988) Initial flow of liquid in an accelerating tank. J. Engng. Mech. 114, 175-180. 